Sebastian Böhlen ${ }^{*}, 1,2$, Sebastian Konzok ${ }^{1,2}$, Jennifer Labisch ${ }^{1,2}$ Susann Dehmel ${ }^{1,2}$, Dirk Schaudien $^{1,2}$, Stephan Behrens ${ }^{3}$, Florian Schmieder ${ }^{3}$, Armin Braun ${ }^{1,2}$ Frank Sonntag ${ }^{3}$, Katherina Sewald ${ }^{1,2}$

\title{
Using a micro-physiological system to prolong the preservation of ex vivo lung tissue
}

\begin{abstract}
Current in vitro and in vivo disease models have been reported to lack sufficient translation to human. Precision-Cut Lung Slices (PCLS) are viable sections of lung tissue and have been described to be a translational model for the ex vivo assessment of pharmacological and toxicological compounds. In most studies PCLS were cultured under static conditions. These lung sections, however, suffer from the limited viability. Here we present a novel modular microphysiological system (MPS) to prolong the cultivation of ex vivo lung tissue. A tailored MPS setup was designed using the PDMS free modular plug\&play MPS construction kit. PCLS from mice were cultivated for up to one week under static versus perfused conditions. Using the MPS technology enabled a prolonged culture period with improved viability as shown by lowered lactate dehydrogenase release and improved membrane integrity. Using this technology might allow us to use PCLS for longer culture periods such as e.g. repeated dose toxicity or pharmacology studies.
\end{abstract}

Keywords: Precision-cut lung slices, organ-on-a-chip, respiratory disease models, long-term cultivation

https://doi.org/10.1515/cdbme-2021-2053

\section{Introduction}

According to the World Health Organization respiratory diseases are one of the leading cause of death besides stroke or heart diseases [11]. Even though, incidences of common respiratory diseases e.g. asthma or chronic obstructive

\footnotetext{
* Sewald, Katherina: Fraunhofer ITEM, Nikolai-Fuchs-Straße 1, Hannover, Germany, katherina.sewald@item.fraunhofer.de 1: Fraunhofer ITEM, Hannover, Germany, Member of the German Center for Lung research (DZL), Biomedical Research in Endstage and Obstructive Lung Disease Hannover (Breath) 2: Fraunhofer Cluster of Excellence Immune-Mediated Diseases (CIMD)

3: Fraunhofer Institute for Material and Beam Technology (IWS), Dresden, Germany
}

pulmonary disease have been increasing over the last decades, development of new therapies remains challenging. One major reason is the lack of appropriate research models to evaluate the safety and efficacy of potential drugs, thereby translating results from non-clinical studies to clinical trials.

Precision-cut lung slices (PCLS) are an useful tool to study respiratory diseases. PCLS are very thin $(100-400 \mu \mathrm{m})$ sections of living lung tissue and maintain the complex, multicellular architecture and microanatomy of the lung [8]. Additionally, they contain resident immune cells such as macrophages and lymphocytes which respond to chemical, physical or biological agents with primary innate and adaptive immune responses $[4,8,10]$. Even functional responses such as airway or vessel constriction can be investigated [7]. Therefore, PCLS overcome some limitations of in vitro systems and are considered as a translational model linking non clinical studies with clinical trials $[6,8]$.

These lung sections, however, suffer from their limited viability. Previous studies showed that human PCLS remain viable for up to four weeks without a loss of their structural integrity. However, loss of cellular / subcellular activities and biological functions can be observed $[6,10]$. Siminski et al. showed that murine PCLS lost their integrity beyond day 7 [9]. Viability differs from species to species. PCLS from mice are less suitable for longer culture periods and show a decrease of viability already after few days [9]. Therefore, a method to prolong the culture period of murine PCLS is urgently needed. Prolonged culture periods might be used for repeated, subchronic or chronic exposure protocols. Other researchers applied approaches such as air-liquid-interface cultures using a foam matrix or a rotating system to improve-long term cultivation of PCLS. In our study, the rapid evolving field of microengineering should be used. Microfluidic systems are reported to better reflect the in vivo situation in regards of nutrient transport and elimination of waste products. Therefore, a microphysiological system used for cultivation of PCLS was developed and tissue viability, histological changes and metabolic activity were analyzed. 


\section{Methods}

\section{Media, reagents and chemicals}

Dulbecco's Modified Eagle's Medium Nutrient Mixture F-12 Ham (DMEM-F12) with L-glutamine, 15 mM HEPES without phenol red, $\mathrm{pH}$ 7.2-7.4, penicillin/streptomycin (P/S), low melting agarose, Earle's Balanced Salt Solution (EBSS) and protease inhibitor cocktail was supplied by Sigma Aldrich (Munich, Germany). Phosphate buffered saline (PBS, without $\mathrm{Ca}^{2+}$ and $\mathrm{Mg}^{2+}$ ) was obtained from Lonza (Wuppertal, Germany). DMEM-F12 was supplemented with $100 \mathrm{U} / \mathrm{mL}$ penicillin and $100 \mu \mathrm{g} / \mathrm{mL}$ streptomycin. LDH cytotoxicity detection kit was purchased from Roche (Mannheim, Germany). Calcein-AM was supplied by ThermoScientific (Dreieich, Germany).

\section{Preparation of PCLS}

PCLS were prepared from mice as described previously [3]. Briefly, mice lungs were inflated with 3\% agarose in DMEM$\mathrm{F} 12$ and cooled to $4^{\circ} \mathrm{C}$. After polymerization of the agarose, tissue was cut into ultrathin slices of $350 \mu \mathrm{m}$ in $4{ }^{\circ} \mathrm{C}$ cold EBSS, using an automatic oscillating microtome (OTS 5000, Warner Instruments, CT, USA). PCLS were kept in DMEMF12 under normal cell culture conditions $\left(37^{\circ} \mathrm{C}, 5 \% \mathrm{CO}_{2}\right.$ and $100 \%$ air humidity). Tissue was intensively three to four times washed in DMEM-F12. For static tissue culture, PCLS were transferred into a 24 well culture plate by placing four PCLS in $500 \mu \mathrm{L}$ DMEM-F12 per well and cultured under normal cell culture conditions $\left(37^{\circ} \mathrm{C}, 5 \% \mathrm{CO}_{2}\right.$ and $100 \%$ air humidity). Control PCLS were lysed with $1 \%$ Triton X-100 for 1 hour as reference for maximum LDH release. $100 \mu \mathrm{L}$ of DMEM-F12 were exchanged each day, with the taken amount being used for the lactate dehydrogenase assay described below.

\section{Biochip technology}

The microfluidic system, as shown in figure 1, is separated into a pumping and a cultivation part. The pumping part consists of the control unit MPScontrol and the triple-pump-chip that is described elsewhere [1,2]. In brief, the triple-pump-chip is made up of three independent channels, each with a cascade of an inlet valve, a pump chamber and an outlet valve. It is connected with the control unit by thin pneumatic hoses. Through frequented change of positive and negative pressured air, regulated by the MPScontrol, the membranes of the chip gets deflected. Thus, the chambers of the pump are charged and discharged with fluid. This initializes a directed pulsatile volume flow. The PCLS-chip that is connected via luer connection tubes (B. Braun SE, Germany) with the triplepump-chip represents the cultivation part. The chip consists of 4 wells $(10 \mathrm{~mm}$ in diameter, $0.75 \mathrm{~mm}$ deep) with an open cultivation tank that can be closed by lid. Via the connection line, the chip is continuously perfused.

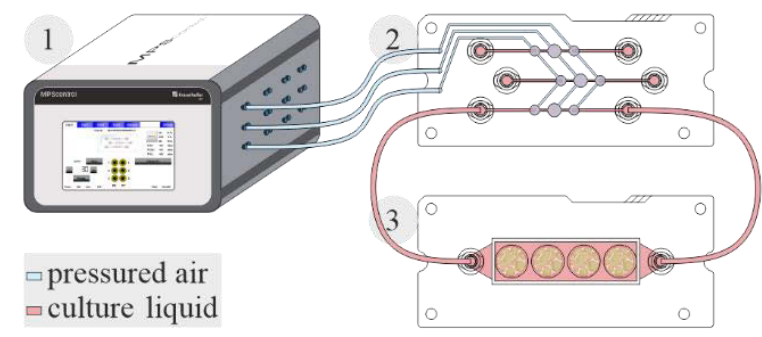

Figure 1: Schematic drawing of the microfluidic setup: 1) pneumatic control module (MPScontrol); 2) triple-pump-chip; 3) PCLS-chip.

\section{PCLS cell culture under dynamically perfused conditions}

The culture chambers of the biochips were sealed with adhesive covers and flushed with $70 \%$ ethanol three times using the pumping system. After subsequent washing of the system with DMEM-F12 for additional six times, the adhesive cover foil was removed and PCLS were placed within the chamber under sterile conditions using an inoculation loop. The chamber with the tissue slices was sealed by a cover slip that was glued on the MPS using picodent twinseal ${ }^{\circledR}$. Biochips were filled with $500 \mu \mathrm{L}$ DMEM-F12, closed with Luer-Locks and cultivated under normal culture conditions $\left(37^{\circ} \mathrm{C}, 5 \%\right.$ $\mathrm{CO}_{2}$ and $100 \%$ air humidity) with a perfusion of about $2.1 \mu \mathrm{L} / \mathrm{s}$. Similar to the immersion culture, $100 \mu \mathrm{L}$ of DMEM were exchanged daily with the taken amount being used for the LDH assay described below.

\section{Cytotoxicity assay}

To determine viability of the slices under different culture conditions, the release of lactate dehydrogenase (LDH) was determined. Therefore, $50 \mu \mathrm{L}$ of tissue culture supernatant were transferred to a 96-well plate in duplicates. LDH reaction mixture were added according to the manufactures instructions and plates were incubated for $20 \mathrm{~min}$ at room temperature, protected from light. Absorbance was measured at $490 \mathrm{~nm}$ with a reference wavelength of $690 \mathrm{~nm}$.

\section{Viability assays}

PCLS were removed from the MPS and cell culture plate, respectively, and were placed in a new 24 well culture plate The working solution of calcein-AM $(4 \mu \mathrm{M})$ was prepared. $250 \mu \mathrm{L}$ of the working solution were added to each well and incubated for $45 \mathrm{~min}$ at room temperature in the dark. Afterwards the staining solution was discarded, PCLS were three times washed with $1 \mathrm{~mL}$ PBS through shaking at $150 \mathrm{rpm}$ 
for $5 \mathrm{~min}$ at room temperature in the dark. $250 \mu \mathrm{L}$ of $1 \%$ Triton-X were added to each well and incubated with tissue for $1 \mathrm{~h}$ at $4{ }^{\circ} \mathrm{C} .100 \mu \mathrm{L}$ of the supernatant were transferred to a new black 96 well plate in duplicates. Fluorescence was measured using plate reader at an excitation wavelength of $495 \mathrm{~nm}$ and an emission at $525 \mathrm{~nm}$.

\section{Hematoxylin and eosin staining of PCLS}

Following fixation in $10 \%$ formaldehyde solution, PCLS were embedded in paraffin blocks and sections of $4 \mu \mathrm{m}$ were performed. On slide of each block was stained with hematoxylin and eosin (H\&E) and slides were evaluated by light microscopy.

\section{Results}

\section{Morphology of lung slices remained intact after one week of microfluidic cultivation}

Lung slices of Balb/c mice (Charles River) were prepared and cultured on a biochip compared to conventional static culture systems for 7 days. H\&E staining of PCLS was performed to analyze the histology of PCLS after seven days of static or fluidic cultivation. Morphologically there was no obvious difference between the PCLS cultivated under fluidic or static condition (Figure 2).

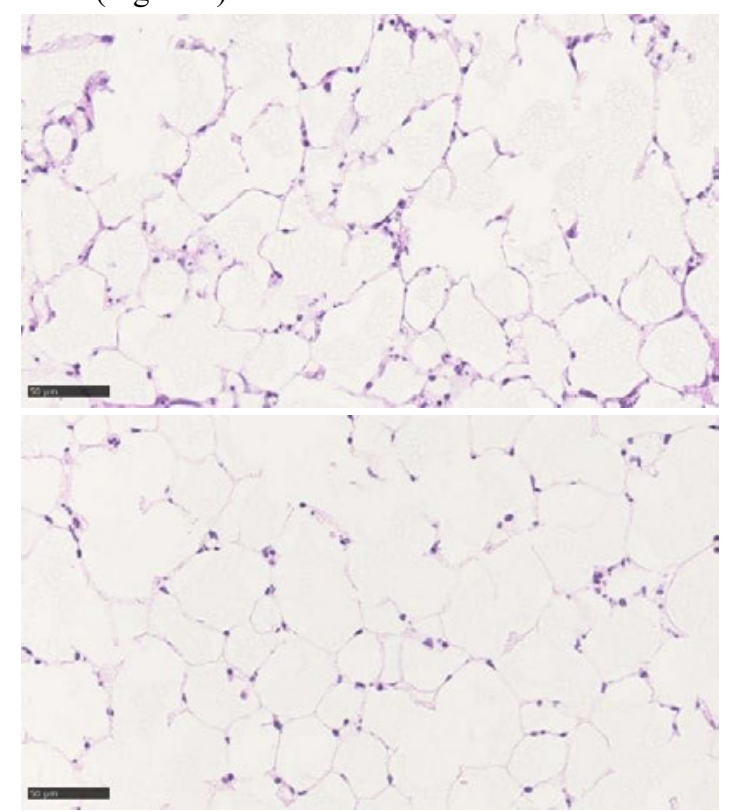

Figure 2: H\&E staining of paraffin embedded murine PCLS which were cultured for up to seven days under static (upper image) or fluidic conditions (lower image). No obvious morphologic difference were observed. Scale bar: $50 \mu \mathrm{M}$

\section{Lung slices showed a prolonged viability under microfluidic conditions}

Viability was assessed by measurement of LDH activity in culture supernatant and of metabolic activity using WST-1 assay. LDH is released from damaged cells and was used to determine the integrity of cell membranes. The LDH release increased over the entire culture period of seven days for both culturing methods. Nearly $70 \%$ of the total LDH were released on day four if the PCLS were cultured under static conditions. Murine PCLS cultured under microfluidic conditions showed lower LDH activity, and hence, increased viability (Figure 3 ).

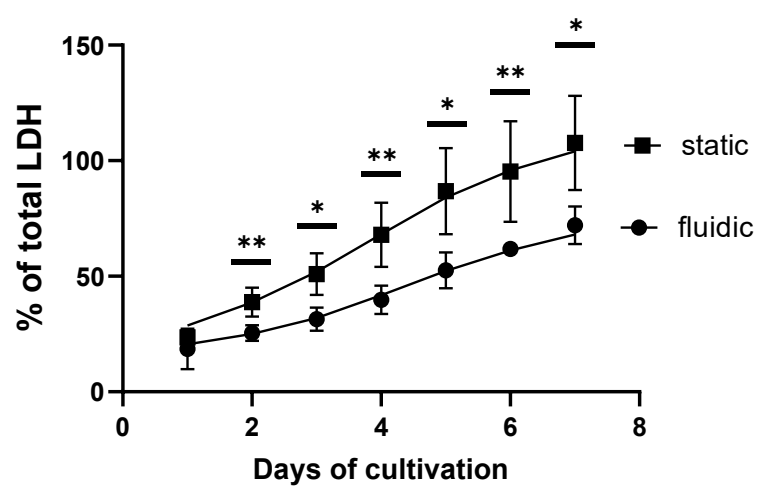

Figure 3: Comparison of viability of PCLS cultured on biochip versus conventional static conditions. PCLS were either culture on biochip $(\bullet)$ or under static conditions ( $\square$ ). Supernatants were frequently taken and used for determination of viability by LDH assay. PCLS lysed with Triton X-100 were used for determination of total LDH. Statically analyses were performed using an unpaired twotailed t-test. P-values are indicated by ${ }^{*}<0.05$ and ${ }^{* *}<0.01$. $\mathrm{n}=5$ day $1-4, \mathrm{n}=4$ day 5 .

As an additional viability assay, the enzymatic activity of the tissue was determined by calcein-AM assay after 3 and 7 days. On day 3 only a slight difference in the enzymatic activity between PCLS cultured under static or fluidic conditions was observed. PCLS on biochip showed a higher enzymatic activity (calcein fluorescence) compared to static conditions on day 7. Static cultivation of PCLS showed an increased variety between donors reaching from non-impacted enzymatic activity to a total loss of the activity whereas PCLS cultured using a MPS showed an enzymatic activity comparable to day 3 (Figure 4). 


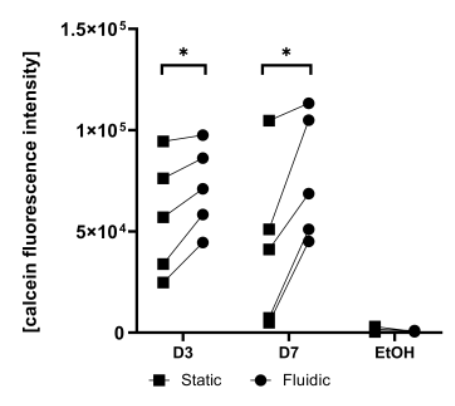

Figure 4: Comparison of enzymatic activity of PCLS. PCLS were either culture on biochip $(\bullet)$ or under static conditions $(\boldsymbol{\square})$.After 3 and 7 days, PCLS were stained using calcein-AM. After staining, PCLS were lysed and supernatants were used for fluorescence intensity measurements. Ethanol treated PCLS served as a dead control. Statically analyses were performed using a paired t-test. P-values are indicated by * $<0.05 ; n=5$

\section{Conclusion}

This study showed that murine lung tissue cultured on biochip/MPS remained viable. Viability was enhanced compared to conventional static conditions. The cultivation period was prolonged from three days up to six days. This improvement might enable longer culture conditions for e.g. repeated dose studies using murine PCLS as an alternative method for in vivo testing. However, further steps are needed to verify that the tissue remains immunocompetent within the MPS and responds to the different mitogens even at later time points.

\section{Author Statement}

Research funding: The authors would like to express great appreciation to the Free State of Saxony and the European Union EFRE (SAB projects "HERMES" and "TRIOMED") as well as the DFG (TRR 305) for the financial support. The project received funding from Fraunhofer grant "MYCELLFIGHT".

Ethical approval: The research related to animals use complied with all the relevant national regulations and institutional policies for the care and use of animals

Conflict of interest: Authors state no conflict of interest.

\section{References}

[1] Behrens, S., Schmieder, F., Polk, C., and Schöps, P. 2021 - 2021. PDMS free modular plug and play construction kit for the development of micro-physiological systems.
In Microfluidics, BioMEMS, and Medical Microsystems XIX. SPIE, 20. DOI=10.1117/12.2585203.

[2] Gray, B. L. and Becker, H., Eds. 2021 - 2021. Microfluidics, BioMEMS, and Medical Microsystems XIX. SPIE.

[3] Henjakovic, M., Martin, C., Hoymann, H. G., Sewald, K., Ressmeyer, A. R., Dassow, C., Pohlmann, G., Krug, N., Uhlig, S., and Braun, A. 2008. Ex vivo lung function measurements in precision-cut lung slices (PCLS) from chemical allergensensitized mice represent a suitable alternative to in vivo studies. Toxicological sciences: an official journal of the Society of Toxicology 106, 2, 444-453.

[4] Martin, C., Uhlig, S., and Ullrich, V. 1996. Videomicroscopy of methacholine-induced contraction of individual airways in precision-cut lung slices. The European respiratory journal 9, 12, 2479-2487.

[5] Nassimi, M., Schleh, C., Lauenstein, H.-D., Hussein, R., Lübbers, K., Pohlmann, G., Switalla, S., Sewald, K., Müller, M., Krug, N., Müller-Goymann, C. C., and Braun, A. 2009. Low cytotoxicity of solid lipid nanoparticles in in vitro and ex vivo lung models. Inhalation toxicology 21 Suppl 1, 104-109. [6] Neuhaus, V., Schaudien, D., Golovina, T., Temann, U.-A., Thompson, C., Lippmann, T., Bersch, C., Pfennig, O., Jonigk, D., Braubach, P., Fieguth, H.-G., Warnecke, G., Yusibov, V., Sewald, K., and Braun, A. 2017. Assessment of long-term cultivated human precision-cut lung slices as an ex vivo system for evaluation of chronic cytotoxicity and functionality. Journal of occupational medicine and toxicology (London, England) 12, 13.

[7] Seehase, S., Switalla, S., Neuhaus, V., Zöller, M., Kaup, F. J., Schlumbohm, C., Fuchs, E., Lauenstein, H. D., Sewald, K., Hohlfeld, J. M., Braun, A., and Knauf, S. 2013. A translational approach to test anti-inflammatory drugs in a LPS induced acute lung inflammation model in the common marmoset (Callithrix jacchus). J Inflamm 10, Suppl 1, P28.

[8] Sewald, K. and Braun, A. 2013. Assessment of immunotoxicity using precision-cut tissue slices. Xenobiotica; the fate of foreign compounds in biological systems 43, 1, 8497.

[9] Siminski, J. T., Kavanagh, T. J., Chi, E., and Raghu, G. 1992. Long-term maintenance of mature pulmonary parenchyma cultured in serum-free conditions. The American journal of physiology 262, 1 Pt 1, L105-10.

[10] Temann, A., Golovina, T., Neuhaus, V., Thompson, C., Chichester, J. A., Braun, A., and Yusibov, V. 2017. Evaluation of inflammatory and immune responses in long-term cultured human precision-cut lung slices. Human vaccines \& immunotherapeutics 13, 2, 351-358.

[11] World Health organization. 2020. The top 10 causes of death. https://www.who.int/news-room/fact-sheets/detail/thetop-10-causes-of-death. Accessed 2 July 2021 